\title{
Bowel obstruction due to Chlamydia trachomatis: a case report and review of literature
}

\author{
Yuta Shibasaki ${ }^{1}$, Makoto Sohda ${ }^{*}$, Hiroomi Ogawa' ${ }^{1}$ Chika Katayama', Naoya Ozawa' , Chika Komine

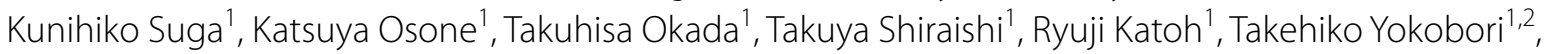 \\ Akihiko Sano ${ }^{1}$, Makoto Sakai ${ }^{1}$, Ken Shirabe ${ }^{1}$ and Hiroshi Saeki ${ }^{1}$
}

\begin{abstract}
Background: Chlamydial infection is a difficult-to-diagnose type of sexually transmitted disease that occurs mainly in young people. We report a case of bowel obstruction caused by intrapelvic adhesions formed by chlamydial infection.

Case presentation: This patient was a 23-year-old woman who had been suffering from acute abdominal pain. She had been previously treated several times for intrapelvic abscesses and had a history of chlamydial infection. Endometriosis was thought to be the cause of her pelvic abscess based on endoscopic findings. Computed tomography demonstrated a small bowel obstruction caused by a pelvic abscess. However, the diagnosis could not be confirmed. She underwent laparoscopic surgery and was diagnosed with bowel obstruction due to adhesion of chlamydial infection based on the intraoperative findings and Chlamydia trachomatis antibody test. She was discharged 5 days after surgery.

Conclusions: It is necessary to consider the possibility of chlamydial infection as a cause for lower abdominal pain and unexplained bowel obstruction in female patients.
\end{abstract}

Keywords: Chlamydia trachomatis, Pelvic inflammatory disease, Bowel obstruction

\section{Background}

Chlamydial infection is a type of sexually transmitted disease (STD) that occurs mainly in young people, and its diagnosis is often difficult due to high frequency of asymptomatic infections [1]. Chlamydial infection can cause pelvic infection disease (PID), ovarian tube abscess, and involves liver capsule inflammation associated with PID [2]. These have been reported as possible causes of adhesion, bowel obstruction, and infertility [2-4]. Although there are few reports of laparoscopic surgery, laparoscopic surgery may be useful in its diagnosis and

\footnotetext{
*Correspondence: msohda@gunma-u.ac.jp

${ }^{1}$ Department of General Surgical Science, Division of Hepatobiliary and Pancreatic Surgery, Graduate School of Medicine, Gunma University, 3-39-22 Showa-Machi, Maebashi, Gunma 371-8511, Japan

Full list of author information is available at the end of the article
}

may make treatment less invasive $[1,2]$. We present a patient who successfully underwent laparoscopic surgery for adherent bowel obstruction due to chlamydial infection.

\section{Case presentation}

This patient was a 23-year-old woman who had acute abdominal pain. She had been treated for intrapelvic abscesses several times previously at another hospital. She also had a history of STD due to chlamydial infection. Computed tomography (CT)-guided drainage was performed, and the patient's symptoms improved. Bloody purulent fluid was noted, and Escherichia coli, but not Chlamydia, was detected by culture test. Polymerase chain reaction (PCR) using urine sample was negative for chlamydial infection when the patient was being treated for pelvic abscesses. In addition, a lower gastrointestinal 
endoscopy was performed to closely examine the source of infection, and intestinal findings suspected endometriosis. Endometriosis is a condition in which endometrium occurs in areas other than the uterus, such as the Douglas fossa, ovaries, intestinal tract, and pelvis. These areas may become infected, resulting in abscess formation. She also had recurrent pelvic abscess, which suggested endometriosis as the cause of the pelvic abscess $[5,6]$. While the patient was being medically treated for endometriosis, she had repeated pelvic abscesses. Thus, she was scheduled to be referred to the obstetrics and gynecology department in our institute for further investigation and treatment.

Meanwhile, she had a relapse of abdominal pain and was taken to our hospital in an emergency. Palpation of the abdomen revealed localized tenderness below the umbilicus and peritoneal irritation symptoms. CT demonstrated a small bowel obstruction with closed loop (Fig. 1a) and a small amount of intrapelvic abscess
(Fig. 1b). Contrast enhancement of the liver surface was also recognized. Laboratory results included white blood cell and platelet counts of $12,800 / \mu \mathrm{L}$ and $224,000 / \mu \mathrm{L}$, respectively. The prothrombin time and international normalized ratio was 0.97 . Total serum bilirubin, albumin, aspartate aminotransferase, alanine aminotransferase, alkaline phosphatase, and C-reactive protein was $1.6 \mathrm{mg} / \mathrm{dL}, 4.3 \mathrm{~g} / \mathrm{dL}, 22,35,239 \mathrm{U} / \mathrm{L}$, and $0.71 \mathrm{mg} / \mathrm{dL}$, respectively (Table. 1).

Taking into consideration the patient's history and clinical findings, the patient was suspected to have bowel obstruction due to pelvic abscesses caused endometriosis infection by Escherichia coli, but the diagnosis could not be preoperatively confirmed.

Laparoscopic surgery was performed for diagnostic and therapeutic purposes. Fibrous adhesions from the liver to the pelvis was intraoperatively recognized. Adhesions in the lower part of the umbilicus formed a small bowel loop, and fibrous adhesions and ascites were also
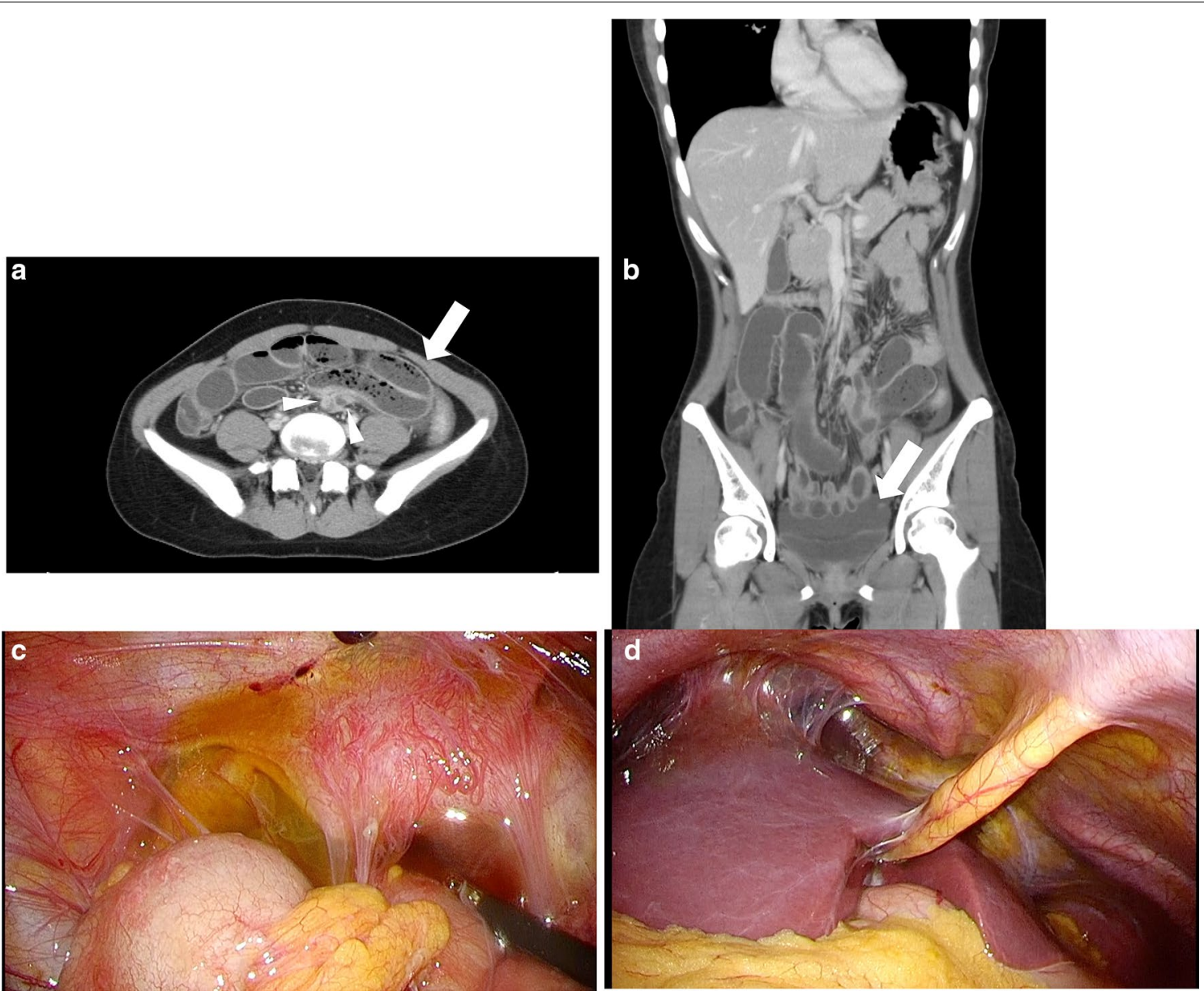

Fig. 1 Abdominal CT and intraoperative findings. a arrow: Closed loop formed and dilated small intestine, arrowheads: Collapsed small intestine on the oral and anal sides of the closed loop, $\mathbf{b}$ ascites in the pelvis, $\mathbf{c}$ ascites in the pelvis and adhesion of the uterine appendages, and $\mathbf{d}$ adhesions that are translucent and considered to be in the active stage of chlamydial infection on the liver surface 
Table 1. Laboratory results

\begin{tabular}{llll}
\hline Blood count & \multicolumn{3}{l}{ Blood chemistry } \\
\hline WBC & $12,800 / \mu \mathrm{L}$ & T-bil & $1.6 \mathrm{mg} / \mathrm{dL}$ \\
Plt & $224,000 / \mu \mathrm{L}$ & Alb & $4.3 \mathrm{~g} / \mathrm{dL}$ \\
& & AST & $22 \mathrm{U} / \mathrm{L}$ \\
& ALT & $35 \mathrm{U} / \mathrm{L}$ \\
Blood coagulation system & ALP & $239 \mathrm{U} / \mathrm{L}$ \\
PT-INR & 0.97 & CRP & $0.71 \mathrm{mg} / \mathrm{dL}$ \\
\hline
\end{tabular}

found in the pelvis (Fig. 1c, d). The uterus could be confirmed, but not the ovaries due to adhesions in the pelvis. Adhesions causing the small bowel loop were found in the small bowel $100 \mathrm{~cm}$ from the Treitz ligament. In addition, multiple other adhesions and bands were found throughout the abdominal cavity. The band due to the adhesions was detached and the bowel obstruction was released. The entire small bowel was then checked and confirmed that there were no other abnormalities. The small bowel was edematous, but not necrotic, and bowel resection was not performed. Intraoperatively, ascitic fluid in the abdominal cavity, including in the pelvis, was aspirated and cleaned as much as possible. There was no obvious abscess formation.

Chlamydia trachomatis antibody IgA was found to be positive, and IgG was negative as shown in the blood sample preoperatively submitted. Based on the typical intraoperative findings and the results of C. trachomatis antibody, she was finally diagnosed with small bowel obstruction caused by the adhesion of chlamydial infection. As for the antibiotics used for treatment, Levofloxacin was used for chlamydial infection. In the perioperative period, Cefmetazole and Clindamycin Phosphate were used for anaerobic infections such as Escherichia coli. Her symptoms improved by adhesion detachment. The postoperative course was uneventful, and the patient was discharged 5 days after surgery.

\section{Discussion}

The strains of C. trachomatis are divided into three biovars and are further subtyped by serovars. Of those, the genital tract biovar (serovars $\mathrm{D}-\mathrm{K}$ ) is associated with the most prevalent STD [7]. In women, $70-80 \%$ of genital tract infections with $C$. trachomatis are asymptomatic, but $15-40 \%$ ascend to the upper genital tract, which can lead to serious sequelae, including PID, tubo-ovarian abscess, perihepatitis (Fitz-Hugh-Curtis syndrome, FHC), infertility, and ectopic pregnancy $[8,9]$.

Also, it has been reported that genital tract infections with $C$. trachomatis can cause adhesions and bowel obstruction $[1,9,10]$. Contrast-enhanced CT shows a characteristically dense subhepatic staining in the early phase. A fibrous adhesion, known as violin string adhesion, is a typical intra-abdominal finding of FHCS [2]. And the positive rate of chlamydial intra-abdominal infection in the presence of violin string adhesion has been reported to be more than $80 \%$ [11]. In our case, we also observed a contrast enhancement of the liver surface on $\mathrm{CT}$ and characteristic intra-abdominal findings such as violin string adhesion on the liver. A slightly purulent ascites in the pelvis was also observed, although the cause was not clear at the point of operation. In this case, IgA was positive and IgG was negative, so the antibody test indicates that the patient was in the active stage of chlamydial infection, which may be early enough for fibrotic adhesions to occur. The intraoperative findings of translucent membranous tissue of perihepatic violin string adhesion and other intra-abdominal adhesions suggest that the patient was still in the active stage. On the other hand, it is also suggested that bowel obstruction due to chlamydial infection can occur even in the active stage, as in this case. Although the possibility that the IgG was false negative and the time of the negative IgG coincided with the early stage of IgA-positive infection were also considered, we comprehensively judged that the bowel obstruction, including the intraoperative findings, was caused by active chlamydial infection [7].

Adhesions (74\%), Crohn's disease (7\%), neoplasia $(5 \%)$, hernia $(2 \%)$, radiation $(1 \%)$, and miscellaneous (11\%), are the etiology of bowel obstruction [12]. Among them, bowel obstruction due to chlamydial infection is extremely rare, and preoperative diagnosis is considered difficult. Bowel obstruction due to chlamydial infection was first described in 1899 , and, so far, only seven cases including our case have been reported in the English literature (Table. 2). Including our case, surgery was performed in five cases for diagnostic and therapeutic purposes, suggesting the difficulty of preoperative diagnosis. On the other hand, two cases improved with conservative treatment with antibiotics and drainage, including a gastric tube $[13,14]$. Only one case obtained preoperative diagnosis as bowel obstruction due to chlamydial infection because the CT showed findings of suspected FHC on the liver surface [3].

As shown in Table 1, laparoscopic surgery has been performed in three cases with bowel obstruction due to chlamydial infection. In one case of the two open surgery, small bowel resection was performed [3, 4]. However, in laparoscopic cases, only a band resection was performed to release the bowel obstruction, and no small bowel resection was performed. Recently, laparoscopic surgery tends to be performed for patients with intestinal obstruction resistant to conservative treatment, especially for with unknown cause [1]. In our case, fibrous 


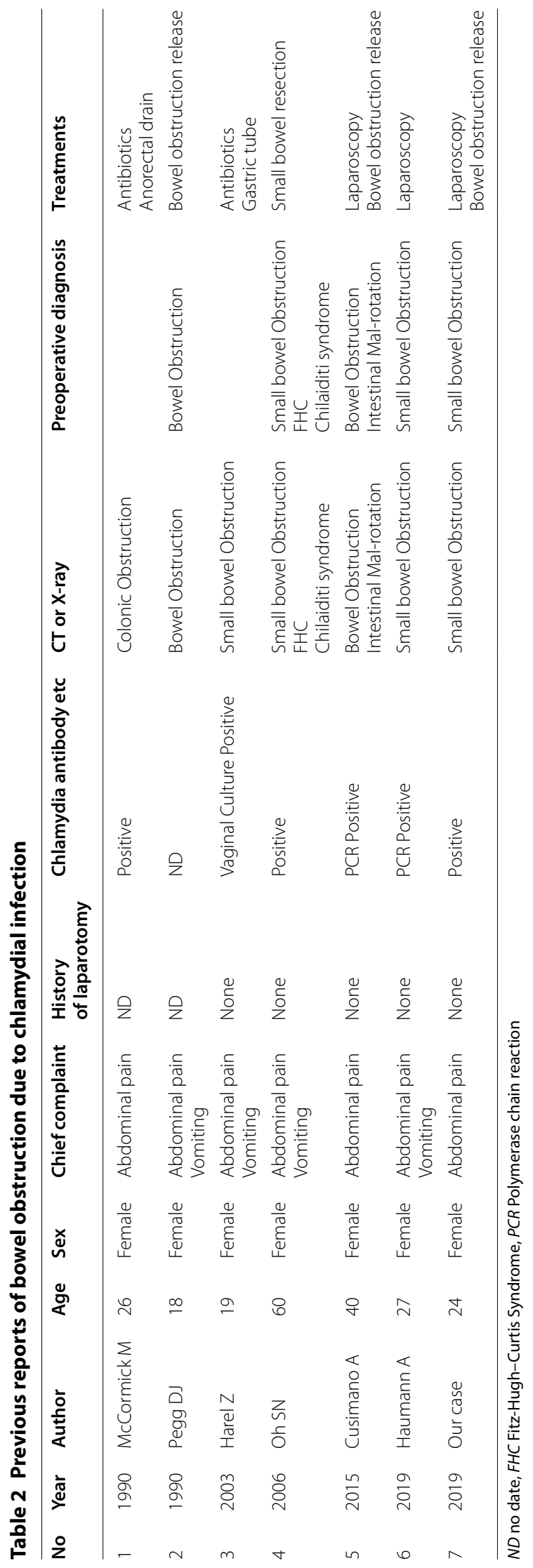


cordage could be dissected by laparoscopic surgery, and the bowel obstruction was resolved. Furthermore, the findings of PID were confirmed in the pelvis and on the liver surface. The advantages of choosing laparoscopic surgery may not only be because of its less invasiveness but also because of its suitability for adequate intraperitoneal observation. The methods of testing for C. trachomatis include cell culture methods using cervical abrasion or urine samples from the affected area, antigen tests (e.g., enzyme immunoassay), genetic tests (e.g., PCR), and serum antibody tests. Cell culture is reported as the standard for testing, but the condition of the facility, the skill, and the cost should be considered. A combination of antigen and genetic tests are generally used to diagnose the current infection [15]. Serum antibody testing should be used as an adjunct because antibodies may not be elevated early in the course of infection [16]. However, serum antibody testing may be useful in cases where specimen collection is difficult (e.g., pelvic infections and pregnant women) and for infertility screening [17]. In our case, neither cell culture from the abscess nor PCRdetected $C$. trachomatis because timing of the chlamydial infection was subclinical. With regard to adhesive bowel obstruction caused by chlamydial infection, a history of infection proven by serum antibody testing, as well as the current infection, may be useful for diagnosis. Both these intraoperative findings and the positivity of C. trachomatis antibody IgA, that was proved postoperatively, led to the diagnosis of bowel obstruction due to chlamydial infection. Testing should be considered to differentiate chlamydial infection for cases with bowel obstructions with unknown cause in women. Chlamydial infection may be suspected, especially in young women, as in this case, or if CT findings indicate that the site of obstruction is slightly above the pelvis.

\section{Conclusions}

We performed laparoscopic surgery for bowel obstruction due to chlamydial infection. Chlamydial infection must be considered for patients with bowel obstruction of undetermined origin.

\section{Abbreviations \\ STD: Sexually transmitted disease; CT: Computed tomography; PID: Pelvic infection disease; FHC: Fitz-Hugh-Curtis syndrome.}

\section{Acknowledgements}

We would like to thank Enago (https://www.enago.jp) for the English language editing.

\section{Authors' contributions}

YS reported the case and wrote the manuscript. HO, CK, NO, CK, KS, KO, TO, TS, $R K, T Y, M S, K S$, and $H S$ performed the surgery and perioperative management of the patient and helped draft the manuscript. HO, TY, MS, KS, and HS participated in revising the manuscript critically. All authors read and approved the final manuscript.
Funding

This study received no funding.

Availability of data and materials

All data generated or analyzed during this study are included in this published article.

\section{Ethics approval and consent to participate}

This case report was performed in accordance with the Declaration of Helsinki, and consent was obtained from the patient to publish according to our institutional consent form.

\section{Consent for publication}

We had consent from the patient to publish.

\section{Competing interests}

The authors declare no competing interest.

\section{Author details}

${ }^{1}$ Department of General Surgical Science, Division of Hepatobiliary and Pancreatic Surgery, Graduate School of Medicine, Gunma University, 3-39-22 Showa-Machi, Maebashi, Gunma 371-8511, Japan. ${ }^{2}$ Innovative Medical Research Center, Gunma University Hospital, Graduate School of Medicine, Gunma University, 3-39-22 Showa-Machi, Maebashi, Gunma 371-8511, Japan.

Received: 24 September 2020 Accepted: 2 February 2021

Published online: 15 February 2021

\section{References}

1. Haumann A, Ongaro S, Detry O, Meunier P, Meurisse M. Acute pelvic inflammatory disease as a rare cause of acute small bowel obstruction. Acta Chir Belg. 2018;119(5):328-30. https://doi.org/10.1080/00015 458.2018.1453438.

2. Cusimano A, Abdelghany AM, Donadini A. Chronic intermittent abdominal pain in young woman with intestinal malrotation, Fitz-Hugh-Curtis Syndrome and appendiceal neuroendocrine tumor: a rare case report and literature review. BMC Women's Health. 2015;16(1):1-8. https://doi. org/10.1186/s12905-015-0274-2.

3. Oh SN, Rha SE, Byun JY, Kim JY, Song KY, Park CH. Chilaiditi syndrome caused by Fitz-Hugh-Curtis syndrome: multidetector CT findings. Abdom imaging. 2006;31(1):45-7. https://doi.org/10.1007/s00261-005-0342-4.

4. Pegg DJ, Owen AW. Gastrointestinal obstruction associated with Chlamydia trachomatis. Genitourin Med. 1990;66(1):26-7. https://doi. org/10.1136/sti.66.1.26.

5. Machairiotis N, Stylianaki A, Dryllis G, et al. Extrapelvic endometriosis: a rare entity or an under diagnosed condition? Diagn Pathol. 2013:8:194. https://doi.org/10.1186/1746-1596-8-194.

6. Koninckx PR, Ussia A, Tahlak M, Adamyan L, Wattiez A, Martin DC, Gomel V. Infection as a potential cofactor in the genetic-epigenetic pathophysiology of endometriosis: a systematic review. Facts Views Vis Obgyn. 2019:11(3):209-16.

7. Meikle SF, Zhang X, Marine WM, Calonge BN, Hamman RF, Betz G. Chlamydia trachomatis antibody titers and hysterosalpingography in predicting tubal disease in infertility patients. Gynecol Endocrinol. 1994;62(2):305-12. https://doi.org/10.1016/S0015-0282(16)56883-6.

8. Elwell C, Mirrashidi K, Engel J. Chlamydia cell biology and pathogenesis. Nat Rev Microbiol. 2016;14(6):385-400. https://doi.org/10.1038/nrmic ro.2016.30.

9. Baumgardner DJ. Abdominal Pain Chlamydia as Culpri. t Postgrad Med. 1989;85(8):281-8. https://doi.org/10.1080/00325481.1989.11700757.

10. Baumgardner DJ. Gastrointestinal obstruction associated with Chlamydia trachomatis. Genitourin Med. 1991;67(1):73. https://doi.org/10.1136/ sti.67.1.73.

11. Hyun JJ, Kim JY, Bak YT, Lee CH, Choi SY. Gastrointestinal: Fitz-Hugh-Curtis syndrome. J Gastroenterol Hepatol. 2006;21(9):1493. https://doi.org/10.11 11/j.1440-1746.2006.04632.x.

12. Miller G, Boman J, Shrier I, Gordon PH. Etiology of small bowel obstruction. Am J Surg. 2000;180(1):33-6. https://doi.org/10.1016/S0002 -9610(00)00407-4. 
13. Harel Z, Tracy TF Jr, Bussey JG III. Small bowel obstruction in an adolescent with pelvic inflammatory disease due to Chlamydia trachomatis. J Pediatr Adolesc Gynecol. 2003;16(3):125-8. https://doi.org/10.1016/S1083 -3188(03)00041-X.

14. McCormick M, Berk RS. An atypical presentation of the Fitz-Hugh-Curtis syndrome. J Emerge Med. 1990;8(1):55-8. https://doi.org/10.1016/0736 4679(90)90388-c.JE.

15. Ozüberk OÖ, Gökahmetoğlu SE, Ozçelik B, Ekmekçioğlu O. Investigation of Chlamydia trachomatis with cell culture, DFA and PCR methods in the genital swab samples of symptomatic patients. Mikrobiyol Bul. 2013;47(1):79. https://doi.org/10.5578/mb.4208.

16. de Haro-Cruz MJ, Guadarrama-Macedo SI, López-Hurtado M, Escobedo-Guerra MR, Guerra-Infante FM. Obtaining an ELISA test based on a recombinant protein of Chlamydia trachomatis. Int Microbiol. 2019;22(4):471-8. https://doi.org/10.1007/s10123-019-00074-4.

17. Muvunyi CM, Dhont N, Verhelst R, Temmerman M, Claeys G, Padalko E. Chlamydia trachomatis infection in fertile and subfertile women in Rwanda: prevalence and diagnostic significance of IgG and IgA antibodies testing. Hum Reprod. 2011;26(12):3319-26. https://doi.org/10.1093/ humrep/der350.

\section{Publisher's Note}

Springer Nature remains neutral with regard to jurisdictional claims in published maps and institutional affiliations.

\section{Submit your manuscript to a SpringerOpen ${ }^{\circ}$ journal and benefit from:}

- Convenient online submission

- Rigorous peer review

- Open access: articles freely available online

- High visibility within the field

- Retaining the copyright to your article

Submit your next manuscript at $\boldsymbol{\nabla}$ springeropen.com 